\title{
THE EFFECT OF THE STRESS STATE ON THE ULTIMATE PLASTICITY OF STEEL X80
}

\author{
D. I. Vichuzhanin ${ }^{1}$, V. A. Khotinov ${ }^{2}$, S. V. Smirnov ${ }^{1}$ \\ ${ }^{I}$ Institute of Engineering Science, Ural Branch of the Russian Academy of Sciences, 34 Komsomolskaya st., \\ Ekaterinburg, Russian Federation \\ ${ }^{2}$ Yeltsin UrFU. Institute of Materials and Metallurgy, 28 Mira st., Ekaterinburg, Russian Federation
}

"Corresponding author. E-mail: mmm@imach.uran.ru ; address for correspondence: ul. Komsomolskaya, 34, 620049, Ekaterinburg, Russian Federation. Tel.: +7 (343) 375-35-96; fax: +7 (343) 374-53-30

The dependence of ultimate plasticity on the triaxiality parameter and the Lode-Nadai coefficient is obtained for steel X80. The fracture locus allows the plasticity of the investigated materials to be evaluated for the whole range of the Lode-Nadai coefficient. Cylindrical specimen tension, bell-type specimen tension and compression and membrane extrusion are used for fracture locus calibration. The results can be used for plasticity resource estimation for steels under a complex stress-strain state in main pipelines subject to extreme loads.

Keywords: high-strength steel, fracture locus, plasticity resource, main pipeline.

\section{References}

1. Morozov Yu.D., Nastich S.Yu., Matrosov M.Yu., Chevskaya O.N. Obtaining high-quality properties of rolled material for large-diameter pipes based on formation of ferrite-bainite microstructure. Metallurgist, 2008, vol. 52, no. 1-2, pp. 21-28.

2. Arabey A.B. Requirements on the metal in gas pipelines. Steel in trans-lation, 2010, vol. 40, no. 7, pp. 601-608.

3. Arabey A.B., Pyshmintsev I.Yu., Farber V.M., Khotinov V.A., Struin A.O. Failure of pipe steel of X80 (K65) strength class. Steel in translation, 2012, vol. 42, no. 3, pp. 212-218.

4. Arabey A.B., Farber V.M., Pyshmintsev I.Yu., Glebov A.G., Selivanova O.V., Lezhnin N.V. Microstructure and dispersed phases in high - strength steels of gas large-diameter pipes. Nauca I technical v gazovoy promishlennosty, 2011, no. 4, pp. 86-91.(In Russian).

5. Rusakova V.V., Lobanova T.P., Arabey A.B., Pyshmintsev I.Yu. Organizing of complex research of domestic pipes for modern gas pipelines of new gen-eration. Nauca I technical $\mathrm{v}$ gazovoy promishlennosty, 2009, no.1, pp. 17-21. (In Russian).

6. Pyshmintsev I.Y., Maltseva A.N., Gervasev A.M., Smirnov M.A., Korznikov A.V. Structure and properties of low - carbon pipe steelafter pneumatic testing. Steel in translation, 2011, vol. 41, no. 2, pp. 157-164.

7. Bridgman P.W. Studies in large plastic flow and fracture. McGraw-Hill, 1952, 362 p.

8. Atkins A.G. Fracture in forming. Journal of Materials Processing Technology, 1996, vol. 56, pp. 609-618.

9. Gubkin S.I. Deformiruemost metallov [Deformability of metals]. Moscow, Metallurgizdat Publ., 1953, 199 p.(In Russian).

10. Smirnov - Alyaev G.A., Rozenberg V.M. Teoriya plastichnykh deformatsyi metallov [Theory of metals plastic deformations]. Moscow, Mashgiz Publ., 1956, 367 p. (In Russian).

11. Bao Y., Wierzbicki T. On fracture locus in the equivalent strain and stress triaxiality space. International Journal of Mechanical Sciences, 2004, vol. 46, iss. 1, pp. 81-98.

12. Achouri M., Germain G., Dal Santo P., Saidane D. Experimental characterization and numerical modeling of micromechanical damage under different stress states. Materials and Design, 2013, vol. 50, pp. 207-222.

Vichuzhanin D. I. et al. / The effect of the stress state on the ultimate plasticity of steel X80 
13. Wierzbicki T., Xue L. On the effect of the third invariant of the stress deviator on ductile frac-ture. Impact \& Crashworthiness Laboratory. Cambridge, MA, MIT Press, 2005, Report 136.

14. Smirnov S.V. Accumulation and healing of damage during plastic metal forming: simulation and experiment.Key Engineering Materials, 2013, vol. 528, pp. 61-69.

15. Gorkunov E.S., Mitropolskaya S.Yu., Vichuzhanin D.I., Tueva E.A. Magnetic methods for estimation of load and damage levels in X70 steel. Physical Mesomechanics, 2011, vol. 14, iss.1-2, pp. 85-93.

16. Cachanov L.M. Time to fracture in creep conditions. Doclady AN SSSR, seriya OTN, 1958, no. 8, pp. 67-75.(In Russian).

17. Rabotnov Yu.N. Voprosy prochnosti materialov $i$ konstruktsyi [Problems of the strength of metals and structures]. Moscow, AN SSSR Publ., 1959, 120 p.(In Russian).

18. Kolmogorov V.L. Napryazhenie, deformatsiya, razrushenie [Stresses, deformation, fracture]. Moscow, Metallurgiya Publ., 1970, 229 p.(In Russian).

19. Bogatov A.A., Mizhiritsky O.I., Smirnov S.V. Resurs plastichnosti metallov pri odrabotke davlenieniem [Metal plasticity resource under metal forming]. Moscow, Metallurgiya Publ., 1984, 144 p.( In Russian).

20. Smirnov S.V., Shveikin V.P. Plastichnost $i$ deformiruemost uglerodistykh stalei pri odrabotke davlenieniem [Plasticity and deformability of carbon steels under metal forming]. Ekaterinburg, YrO RAN Publ., 2009, 256 p.( In Russian).

21. Cockcroft M.G., Latham D.J. Ductility and the workability of metals. Journal of the Institute of Metals, 1968, vol. 96, pp. 33-39.

22. Gurson A.L. Continuum theory of ductile rupture by void nucleation and growth: Part I. Yield critetria and flow rules for porous ductile media. Journal of Engi-neering Materials and Technology, 1977, vol. 99, pp. 2-15.

23. Johnson G.R., Cook W.H. Fracture characteristics of three metals subjected to various strains, strain rates, temperatures and pressures. Engineering Fracture Mechanics, 1985, vol. 21 (1), pp. 31-48.

24. Lemaitre J. A Course on Damage Mechanics. Berlin, Springer-Verlag, 1992. 44 p.

25. Bai Y., Wierzbicki T. A new model of metal plasticity and fracture with pressure and Lode dependence. International Journal of Plasticity, 2008, vol. 24, iss. 6, pp. 1071-1096.

26. Bammann D.J., Solanki K.N. On kinematic, thermodynamic, and kinetic coupling of a damage theory for polycrystalline material. International Journal of Plasticity, 2010, vol. 26, iss. 6, pp. 775-793.

27. Davidenkov N.N., Spiridonova N.I. Mechanical methods of testing analysis of the state of stress in the neck of a tension test specimen. Proc. ASTM, 1946, vol. 46, p. 1147.

28. Levanov A.N., Kolmogorov V.L., Burkin S.P. Kontaktnoe trenie v protsessakh obrabotki metallov davleniem [Contact friction in metal forming processes]. Moscow, Metallurgiya Publ., 1976, 416 p.(In Russian).

29. Smirnov S.V., Domilovskaya T.V., Bogatov A.A. In: Advanced Methods in Materials Processing Defects, by M. Predeleanu and P. Gilormini. Elsivier Science B.V, 1997, pp. 71-80.

30. Hooke R., Jeeves T. A Direct Search Solution of Numerical and Statistical Problems. Journal of the ACM, 1961, vol. 8, pp. 212-229.

31. Islamgaleeva L.F., Zaripov R.M. [Influence of extent of ground flooding of adjacent un-derground sections on stress - strain state of underwater gas pipeline] Electronnyi nauchny zhurnal «Neftegazovoe delo», 2011, no. 6 (In Russ.) Available at: http://www.ogbus.ru/authors/Islamgaleeva/Islamgaleeva_1.pdf (accessed 21.07.2014).

32. Suzuki N., Kondo J., Shimamura J. Strain Capacity of High - Strength Line Pipes. JFE Technical Report, 2008, no. 12, pp. 20-26. 


\title{
ВЛИЯНИЕ НАПРЯЖЕННОГО СОСТОЯНИЯ НА ПРЕДЕЛЬНУЮ ПЛАСТИЧ- НОСТЬ ТРУБНОЙ СТАЛИ ГРУППЫ ПРОЧНОСТИ Х80
}

\author{
Д. И. Вичужанин ${ }^{1}$, В. А. Хотинов ${ }^{2}$, С. В. Смирнов ${ }^{1}$ \\ ${ }^{1}$ Федеральное государственное бюджетное учреждение науки Институт машиноведения Уральского отделе- \\ ния Российской академии наук, ул. Комсомольская, 34, Екатеринбург, Российская Федераџия \\ ${ }^{2}$ ФГАОУ ВПО «УрФУ имени первого Президента России Б.Н.Ельична.» Институт материаловедения и ме- \\ таллургии, ул. Мира, 28, Екатеринбург, Российская Федерация \\ * Ответственный автор. Электронная почта: $\mathrm{mmm} @$,imach.uran.ru ; Адрес для переписки: ул. Комсомольская, 34, \\ Екатеринбург, Российская Федерация; Телефон: +7 (343) 375-35-96; Факс: +7 (343) 374-53-30
}

Получена зависимость предельной пластичности стали группы прочности X80 от показателя напряженного состояния и показателя Лоде - Надаи. Диаграмма позволяет оценивать пластичность исследуемых материалов во всем интервале изменения показателя Лоде Надаи. Для построения диаграммы пластичности использовались испытания на растяжение гладких цилиндрических образцов и образцов с выточкой, растяжение и сжатие образцов типа «колокольчик», выдавливание мембран. Результаты исследований могут быть использованы для оценки ресурса пластичности сталей в условиях сложного напряженнодеформированного состояния при экстремальных нагрузках магистральных трубопроводов.

Ключевые слова: высокопрочные стали, диаграмма пластичности, ресурс пластичности, магистральный трубопровод.

\section{1. Введение}

Магистральные трубопроводы могут эксплуатироваться в различных порой экстремальных климатических условиях. При этом основными факторами, влияющими на напряженно-деформированное состояние, являются внутреннее давление и продольные усилия, вызванные перепадом напряжений в линейной части трубопровода вследствие различия температурных условий строительства и эксплуатации. Также не исключены и деформации, связанные с подвижностью грунта, непроектными нагружениями в условиях паводков и т. п. Поэтому в процессе эксплуатации условия термомеханического воздействия на трубу магистрального трубопровода могут изменяться в широком диапазоне значений. В связи с вышесказанным большое значение имеет оценка деформационных свойств и ресурса пластичности основного металла труб и сварных соединений трубопровода, накопленной в процессе эксплуатации.

Разработка новых сталей и использование современных технологий позволили получать высокопрочный лист и, соответственно, сварные трубы с исключительно высоким уровнем вязко-пластических характеристик $[1,2]$. Так, стали класса прочности Х80 обладают ультрамелкозернистой структурой феррита с дозированным ( 30\%) количеством бейнита/мартенсита, формирующейся в горячекатаном листе по технологии контролируемой прокатки и последующего ускоренного охлаждения. Такая структура обеспечивает им крайне высокий уровень ударной вязкости $\mathrm{KCV} \approx 3,0$ МДж/м² вплоть до низких температур испытания $\mathrm{t}_{\text {исп }}=-40{ }^{\circ} \mathrm{C}[3,4]$. Однако в условиях испытаний на ударную вязкость материал исследуется в конкретных условиях напряженно-деформированного состояния, которые в полной мере не реализуются при эксплуатации трубопровода. Проведение полномасштабных полигонных (натурных) испытаний $[5,6]$ осуществляется в условиях, близких к условиям эксплуатации, однако требуют существенных затрат. Кроме того, в этом случае не учитывается исчерпание ресурса пластичности сталей при термо-упруго-пластических статических и цик-

Vichuzhanin D. I. et al. / The effect of the stress state on the ultimate plasticity of steel X80 
лических деформациях, инициирующих ускорение процесса возникновения и развития трещин. Поэтому в данной статье сделана попытка оценить деформационную способность материала трубы и поврежденность в широком диапазоне изменений напряженнодеформированного состояния путем использования простых лабораторных экспериментов.

На деформационную способность материала могут влиять различные параметры напряженно-деформированного состояния. Так, в работах [7-11] было показано существенное влияние гидростатического давления. В работах [12-15] деформационная способность материала рассматривалась также в зависимости от вида напряженного состояния. В качестве критерия, позволяющего оценить деформационную способность материала, может быть использована величина поврежденности. Впервые кинематические уравнения накопления поврежденности были предложены Качановым [16] и Работновым [17].

Феноменологические модели поврежденности при больших пластических деформациях были разработаны В.Л. Колмогоровым и представителями его научной школы [18-20]. За рубежом развитием моделей поврежденности занимались такие ученые, как Кокрафт, Латам, Гарсон, Джонсон, Кук, Ламатр, Вержбицкий и др. [21-26].

В соответствии с феноменологической теорией под поврежденностью понимается скалярный параметр $\omega$ [18-20], который изменяется от 0 в начальный момент деформации до 1 при разрушении. В общем случае можно выделить два критических значения поврежденности $\omega^{*}=0,2-0,4$ и $\omega^{* *}=0,6-0,8$. Электронно-микроскопические исследования показали, что достижение поврежденностью значения $\omega^{*}$ связано с образованием энергетически устойчивых микропор, а $\omega^{* *}-$ с коалесценцией изолированных микропор и образованием микродефектов в виде пор и микротрещин [20]. Значения $\omega^{*}$ и $\omega^{* *}$ не одинаковы для разных материалов. В соответствии с теорией повреждённость $\omega$ в условиях монотонной деформации может быть определена по формуле:

$$
\omega=\int_{0}^{\Lambda} \frac{d \Lambda}{\Lambda_{p}}
$$

где $d \Lambda=\int_{0}^{t} H d \tau$ - степень деформации сдвига; $H$ - интенсивность скоростей деформации сдвига; $\Lambda_{p}\left(k, \mu_{\sigma}\right)$ - пластичность [18], определяемая как предельная степень деформации сдвига, накопленная образцом к моменту разрушения при механических испытаниях в условиях монотонного деформирования при постоянных на всем процессе деформирования показателя напряженного состояния $k$ и показателя Лоде-Надаи $\mu_{\sigma}$ :

$$
k=\frac{\sigma}{T} ; \mu_{\sigma}=2 \frac{\sigma_{22}-\sigma_{33}}{y_{11}-\sigma_{33}}-1,
$$

где $\sigma=\frac{1}{3}\left(\sigma_{11}+\sigma_{22}+\sigma_{33}\right)$ - среднее нормальное напряжение; $T=\sqrt{0,5 S_{i j} S_{i j}}-$ интенсивность касательных напряжений; $S_{i j}-$ компоненты девиатора напряжений $\sigma_{11}, \sigma_{22}, \sigma_{33}-$ главные напряжения. Совокупность показателей $k$ и $\mu_{\sigma}$ однозначно характеризует напряженное состояние при пластической деформации, кроме того, являясь инвариантными и безразмерными, они позволяют сопоставлять напряженное состояние материалов с различным уровнем прочностных свойств. Графическая интерпретация функции $\Lambda_{p}\left(k, \mu_{\sigma}\right)$ называется диаграммой пластичности [18].

Цель данной работы - построение диаграммы предельной пластичности исследуемой стали группы прочности X80. В дальнейшем, промоделировав процесс нагружения трубопровода и воспользовавшись диаграммой пластичности, можно оценить поврежденность материала трубопровода. Также возможно сравнение деформационных свойств различных материалов путем сравнения их диаграмм пластичности. 


\section{2. Материал и методика}

В качестве материала для исследования использовалась низкоуглеродистая сталь группы прочности $\mathrm{X} 80$, содержащая $0,07 \% \mathrm{C}, \sim 2 \% \mathrm{Mn}$ с содержанием $\mathrm{V}+\mathrm{Nb}+\mathrm{Ti} \geq 0,15 \%$. Данная сталь применяется для сварных труб большого диаметра (1420 мм) с толщиной стенки 27,7 мм, изготовленных из листа, полученного по режиму контролируемой прокатки с ускоренным охлаждением. Для определения механических свойств стали проводились испытания пятикратных цилиндрических образцов диаметром 5 мм на растяжение на испытательной установке INSTRON 8801. Скорость испытания составила 1 мм/мин. Сопротивление деформации $\sigma_{S}$ до момента начала образования шейки определяли по формуле:

$$
\sigma_{S}=\frac{P}{F}
$$

где $P$ - текущее усилие нагружения; $F$ - текущая площадь поперечного сечения образца. После образования шейки сопротивление деформации определяли с учетом поправки Давиденкова-Спиридоновой на трехосность напряженного состояния в шейке [27]:

$$
\sigma_{S}=\frac{P}{F\left(1+\frac{d}{8 R}\right)}
$$

где $d / R$ - безразмерный параметр, характеризующий форму шейки образца; $d$ - текущий минимальный диаметр образца; $R$ - текущий минимальный радиус кривизны образующей в шейке. Величины $d$ и $R$ определяли в процессе испытания при помощи бесконтактного оптического комплекса для анализа формы, полей перемещений и деформаций StrainMaster.

Величину степени деформации $\varepsilon$ при растяжении определяли по формуле:

$$
\varepsilon=2 \ln \left(d_{0} / d\right)
$$

где $d_{0}$ - начальный диаметр образца. По результатам расчетов кривая упрочнения была аппроксимирована степенной зависимостью

$$
\sigma_{S}=580+412 \varepsilon^{0,37}(\mathrm{M \Pi а}) .
$$

Для определения функции $\Lambda_{p}=\Lambda_{p}\left(k, \mu_{\sigma}\right)$ были проведены испытания на растяжение гладких цилиндрических образцов и цилиндрических образцов с выточкой, испытания на растяжение и сжатие образцов типа «колокольчик», выдавливание мембран. Исследования выполняли на испытательной установке INSTRON 8801. Скорость испытания составила 1 мм/мин. Использование различных видов испытаний позволяет в достаточно широком диапазоне варьировать показатель напряженного состояния $k$ и показатель Лоде-Надаи $\mu_{\sigma}$.

\section{3. Результаты и обсуждение}

\section{1 Испытания на растяэение цилиндрических образцов}

На данном этапе исследований проведены испытания на растяжение гладких цилиндрических образцов и образцов с выточкой. В данном виде испытаний показатель $\mu_{\sigma}=-1$ на протяжении всего процесса испытания.

Гладкие цилиндрические образцы имели следующие размеры: длина рабочей части $25 \pm 0,1$ мм; диаметр рабочей части $5 \pm 0,06$ мм. Диаметр образцов с выточкой соответствовал 8 мм, радиус выточки в продольном направлении 2,5 $\pm 0,06$ мм, диаметр в месте выточки $5 \pm 0,06$ мм.

Vichuzhanin D. I. et al. / The effect of the stress state on the ultimate plasticity of steel X80 
Изменение показателя $k$ при растяжении рассчитывали по формуле [20]:

$$
k=\frac{1}{\sqrt{3}}\left(1+\frac{3}{4} \frac{d}{R}\right) .
$$

Значения текущего диаметра $d$ и продольного радиуса выточки $R$ в процессе испытания определяли при помощи бесконтактного оптического комплекса StrainMaster. B pезультате были получены зависимости изменения показателя напряженного состояния $k$ в процессе испытания вплоть до разрушения (рис. 1). Предельная степень деформации сдвига $\Lambda$ при разрыве гладкого образца составила 2,25, а образца с выточкой - 2,04.

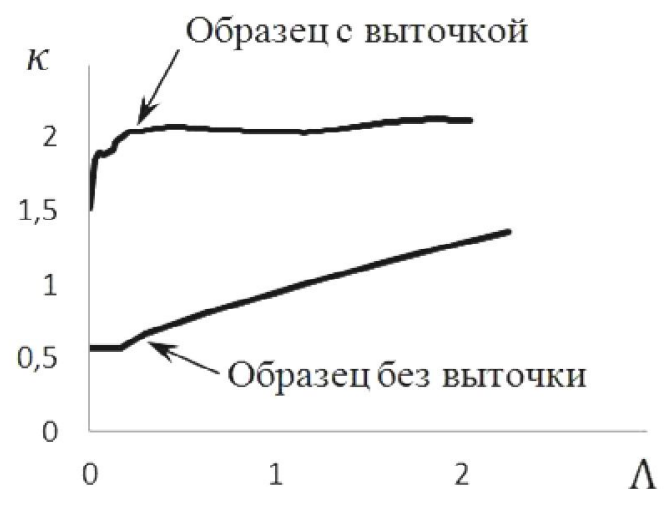

Рис. 1. Изменение показателя напряженного состояния $k$ в процессе деформации цилиндрических образцов

\section{2 Испытания на растяжение и сжатие образцов типа «колокольчик»}

Известно, что существует определенная проблема исследования пластичности при постоянном показателе $\mu_{\sigma}=0$. Для исследований могут использоваться испытания на кручение цилиндрических образцов. Однако этот вид испытаний не позволяет варьировать величину показателя напряженного состояния $k$. Получить значения показателя $k$ в отрицательном интервале значений можно путем приложения к образцу высоких гидростатических давлений [20]. Техника высоких давлений достаточно сложна в эксплуатации. В работе [11] предложен образец, позволяющий производить испытания в широком диапазоне изменения показателя напряженного состояния при постоянном показателе $\mu_{\sigma}=0$. Для испытаний образца необходимо использовать испытательную машину сложной конструкции с двумя исполнительными органами.

Поэтому был предложен образец типа «колокольчик» (рис. 2 a), позволяющий в широком диапазоне варьировать значения показателя напряженного состояния $k$ при неизменном показателе $\mu_{\sigma}=0$. В случае испытаний данного вида образцов на растяжение в месте разрушения преобладают растягивающие напряжения, в случае испытаний на сжатие - сжимающие. Разрушенные образцы показаны на рис. 26 и 2 в (для наглядности образцы разделены вдоль оси симметрии).

Напряженно-деформированное состояние в образце оценивалось по результатам моделирования процесса испытаний методом конечных элементов с использованием пакета ANSYS v. 14.0. Расчеты выполнялись на вычислительном кластере «Уран» института ИММ УрО РАН. Для материала образца принималась изотропная упруго-пластическая модель деформационного упрочнения. Расчеты были выполнены в предположении осесимметричного деформированного состояния в очаге деформации, поэтому моделировалась только половина продольного сечения образца. 
На рис. 3 приведено распределение эквивалентных пластических деформаций $\varepsilon_{e q}$ по сечению образца в процессе испытаний на растяжение и сжатие к моменту разрушения. Величину $\varepsilon_{e q}$ рассчитывали по формуле

$$
\varepsilon_{e q}=\sum_{n}\left(d \varepsilon_{e q}\right)_{n}
$$

где $d \varepsilon_{e q}=\left(\frac{2}{3} d \varepsilon_{i j} d \varepsilon_{i j}\right)^{1 / 2}, n-$ количество шагов расчета.

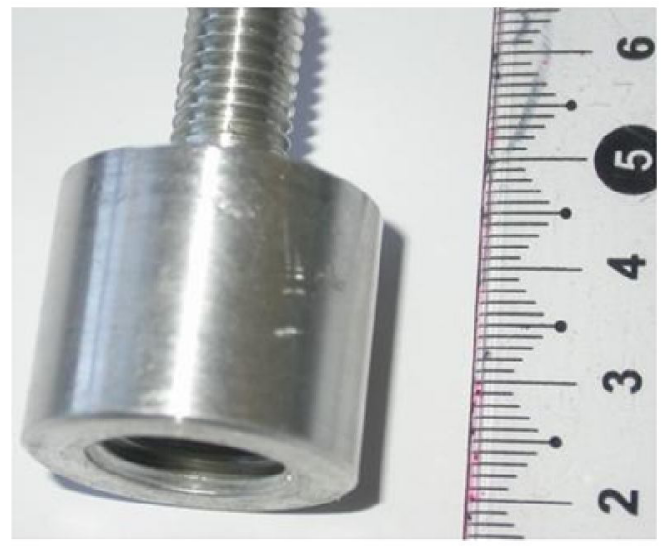

$a$

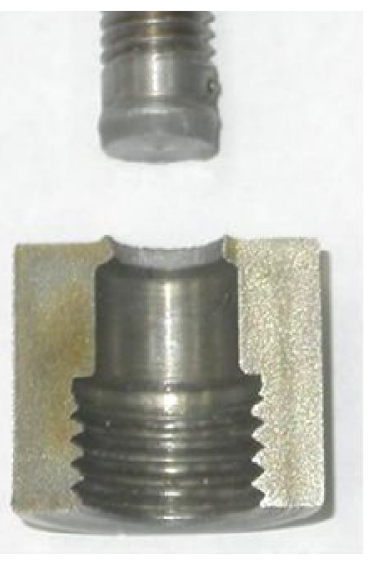

$\sigma$

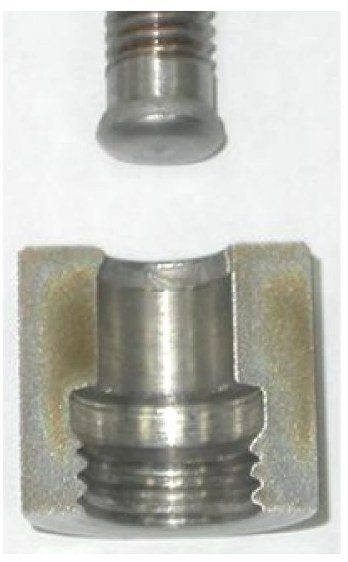

B

Рис. 2. Образец типа «колокольчик»: $a$ - до испытания; $\sigma$ - после испытания на растяжение; в - после испытания на сжатие

По результатам моделирования установлено, что в месте разрушения величина показателя $\mu_{\sigma}=0$ и практически не изменяется на протяжении всего процесса деформирования. Изменение показателя $k$ в процессе испытаний вплоть до момента разрушения представлено на рис. 4. Данные на рис. 4 были получены в местах разрушения (см. рис. 3). Адекватность результатов моделирования оценивалась путем сравнения расчетного усилия деформирования, с экспериментальными данными. Отклонение расчетных и экспериментальных данных не превышало $7 \%$. Значение предельной деформации сдвига $\Lambda$ в месте разрушения для испытания на растяжение составило 1,74, а для испытания на сжатие - 3,44.

\section{3 Выдавливание мембран}

Для проведения испытаний был разработан образец (рис. 5) и оснастка к испытательной машине INSTRON 8801. Для снижения трения между образцом и деформирующим инструментом в донышке образца имеется углубление, в которое помещается смазка в виде порошкообразного графита. Перед началом испытаний образец 1 устанавливается на опору 2 (рис. 6). Сверху образец зажимается крышкой 3. В случае, когда необходимо проводить испытания в условиях преобладающих сжимающих напряжений, под мембраной устанавливался изготовленный из материала мембраны подпор 4, который также деформируется в процессе испытания. Снизу подпор поджимается винтом 5. В качестве деформирующего инструмента использовался шарик 6 диаметром 9,5 мм. Деформирующее усилие на шарик передавалось через пуансон 7, установленный в захвате испытательной машины. Испытания проводились до появления трещины на образце (рис. 7). При испытаниях с подпором разрушение мембраны происходило не в центре, а на некотором расстоянии от него (рис. 8).

Напряженно-деформированное состояние в мембране оценивали по результатам математического моделирования процесса методом конечных элементов в пакете ANSYS.

Деформирующий инструмент (шарик) в модели принимали как абсолютно жесткое тело. Для деформируемого материала мембраны и подпорки принимали изотропную упруго-

Vichuzhanin D. I. et al. / The effect of the stress state on the ultimate plasticity of steel X80 
open-access journal

пластическую модель деформационного упрочнения. Расчеты были выполнены в предположении осесимметричного деформированного состояния в очаге деформации, поэтому моделировали только половину сечения образца и подпорки.

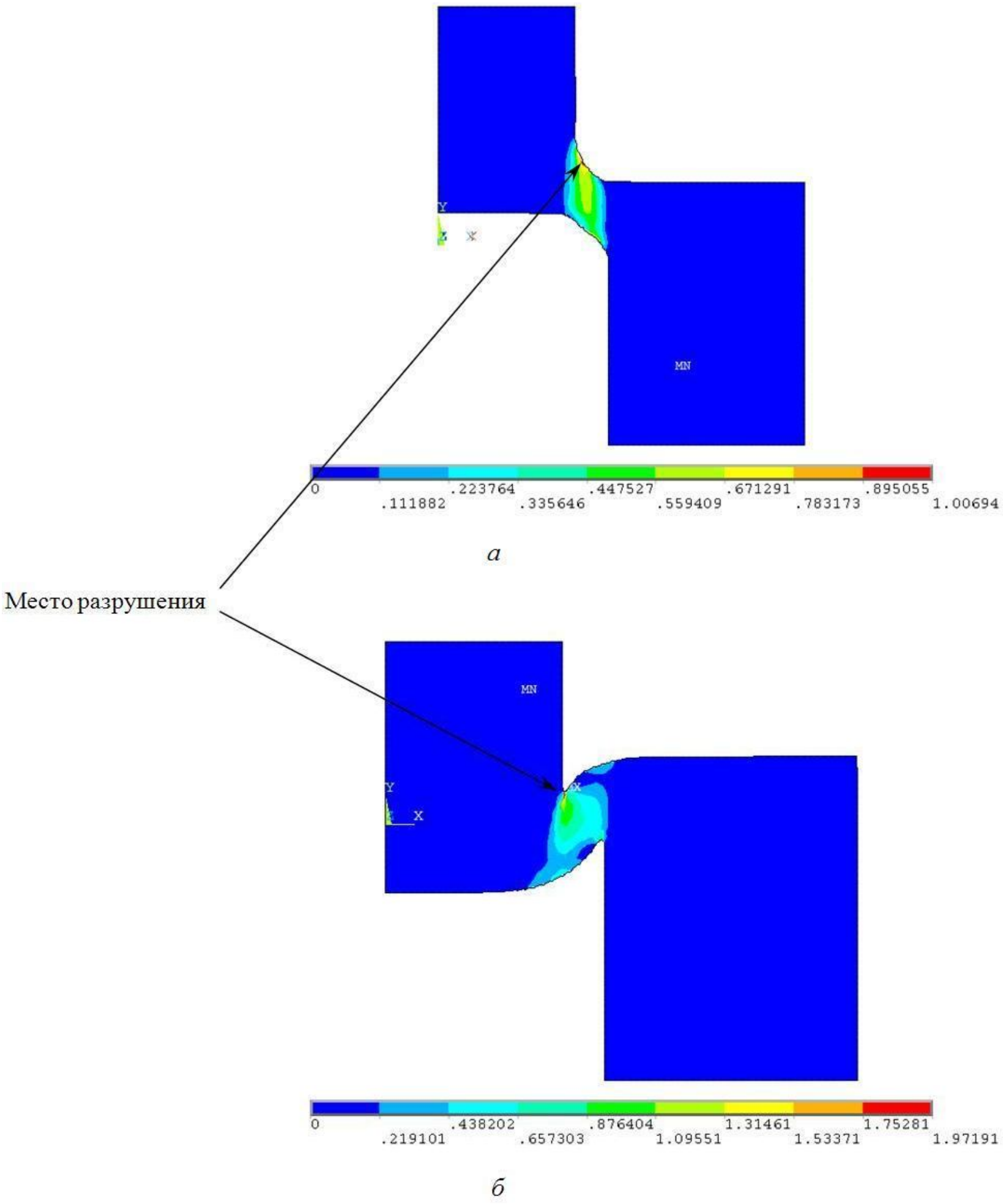

Рис. 3. Распределение эквивалентных пластических деформаций по сечению образца при испытаниях на растяжение ( $a$ ) и на сжатие (б). 
На контактных поверхностях напряжения трения описывали, используя закон трения А.Н. Леванова [28]:

$$
\tau=K_{\Pi} \frac{\sigma_{\mathrm{S}}}{\sqrt{3}}\left[1-\exp (-1,25) \frac{\mathrm{p}}{\sigma_{\mathrm{S}}}\right]
$$

где $K_{\Pi}$ - фактор трения; $\mathrm{p}$ - нормальное контактное давление; $\sigma_{\mathrm{S}}$ - сопротивление деформации в приконтактном слое. Закон трения А.Н. Леванова был выбран в связи с тем, что закон трения Амонтона-Кулона, используемый в ANSYS, завышает напряжения трения в условиях значительных контактных давлений. Эти условия возникают при выдавливании мембран с противодавлением. Закон трения А.Н. Леванова является обобщением законов АмонтонаКулона и Зибеля.

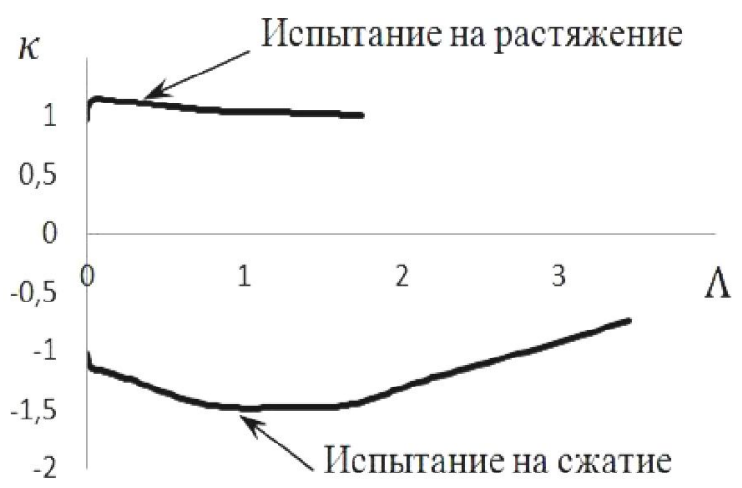

Рис. 4. Изменение показателя напряженного состояния $k$ в процессе деформации образцов типа «колокольчик»

При низких контактных давлениях напряжения трения по А.Н. Леванову близки к напряжениям трения по Амонтону-Кулону, а при высоких контактных давлениях - по Зибелю. Задание закона трения (9) осуществляли с помощью пользовательской подпрограммы USERFRIC. Фактор трения $K_{\Pi}=0,05$ определяли в соответствии с методикой [28] по бочкообразованию цилиндрических образцов при осадке.

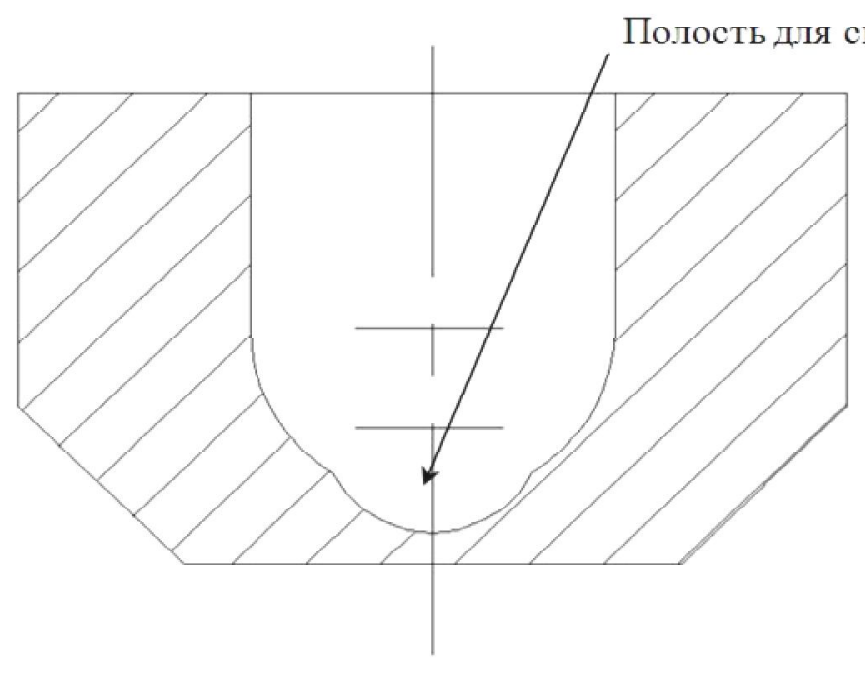

$a$

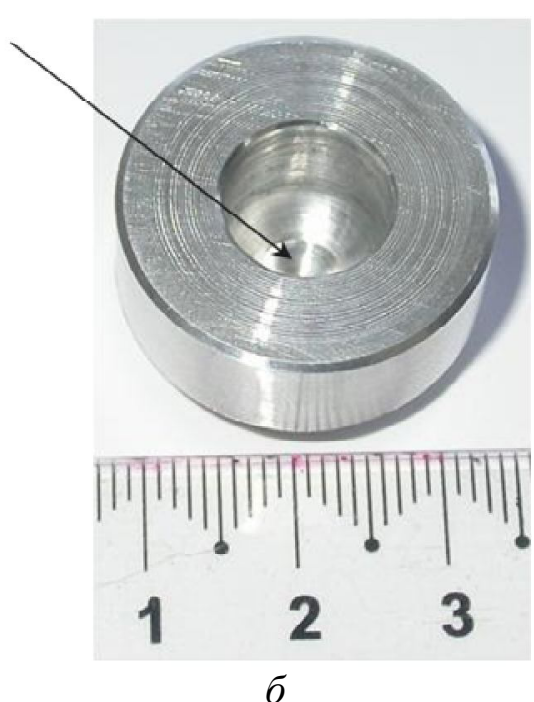

б

Рис. 5. Образец «мембрана»: $a$ - продольное сечение; $\sigma$ - натурный образец

На рис. 9 приведено распределение эквивалентных пластических деформаций по сечению образца к моменту разрушения. По результатам моделирования было установлено из-

Vichuzhanin D. I. et al. / The effect of the stress state on the ultimate plasticity of steel X80 
менение показателей $k$ и $\mu_{\sigma}$ (рис. 10) для участков образцов, где в испытаниях было зафиксировано возникновение трещины. Адекватность полученных при моделировании результатов оценивалась путем сравнения усилия деформирования, полученного при моделировании с экспериментальными данными. Отклонение расчетных и экспериментальных данных не превышает $8 \%$. Значение предельной деформации сдвига $\Lambda$ в месте разрушения для испытания на выдавливание мембраны без подпора составило 1,41, а для испытания на выдавливание с подпором - 2,32.

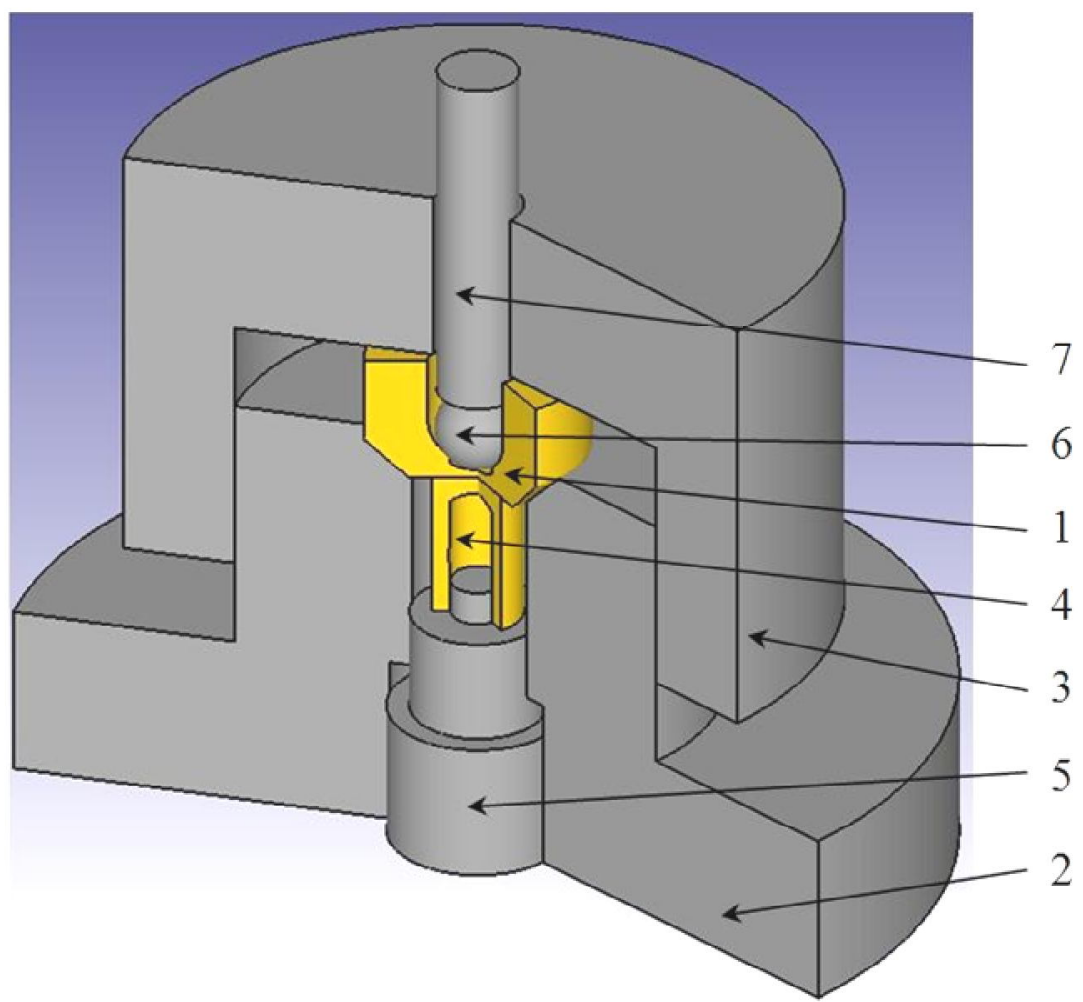

Рис. 6. Схема оснастки для выдавливания мембран

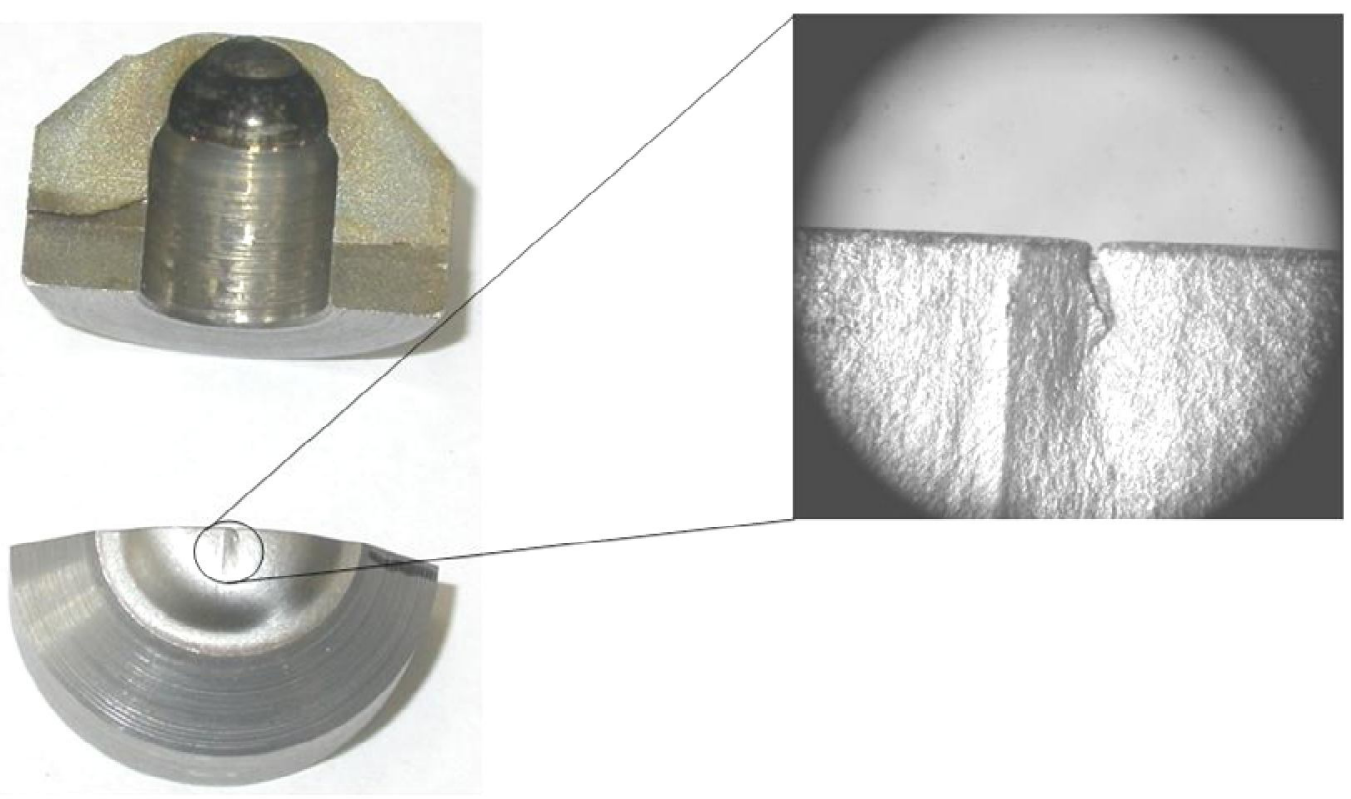

Рис. 7. Образец после испытаний без подпора

Vichuzhanin D. I. et al. / The effect of the stress state on the ultimate plasticity of steel X80 


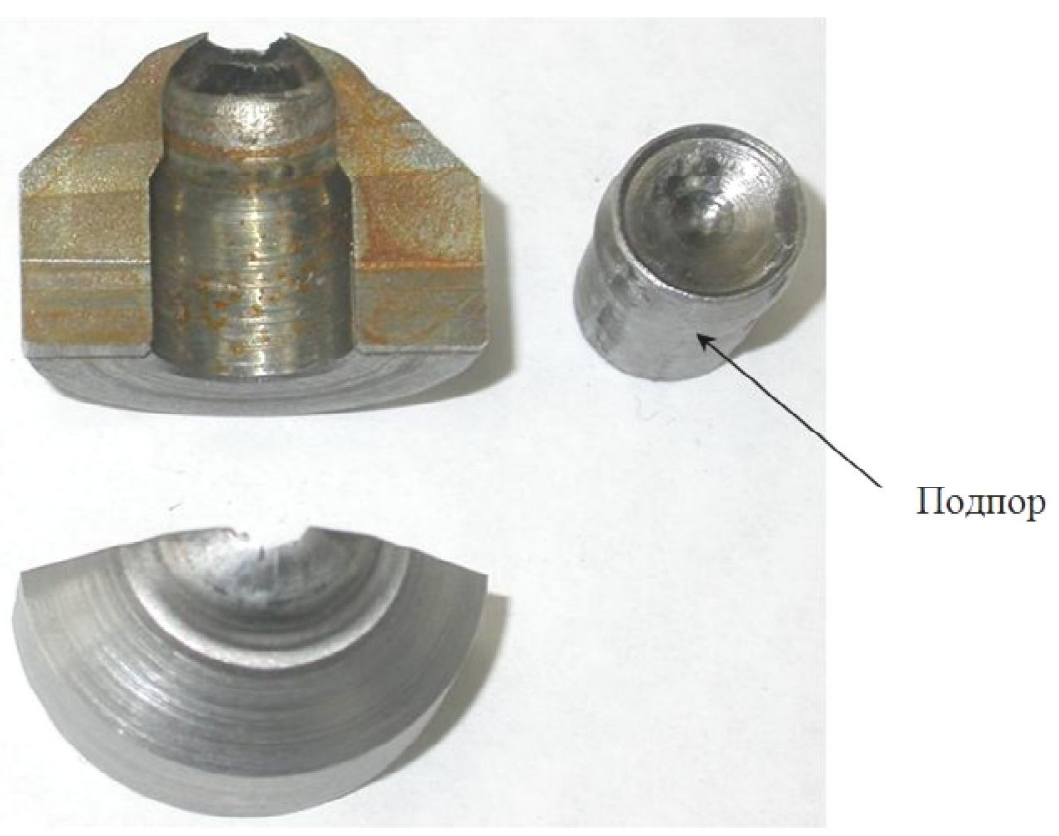

Рис. 8. Образец после испытаний с подпором

\section{4 Идентификация диаграммы пластичности}

Функция, определяющая диаграмму пластичности, в общем случае зависит от двух показателей $-k$ и $\mu_{\sigma}$ и может быть аппроксимирована формулой [19]:

$$
\Lambda_{p}=a_{0}(k)+a_{1}(k) \cdot \mu_{\sigma}+a_{2}(k) \cdot \mu_{\sigma}^{2},
$$

где $a_{0}(k), a_{1}(k), a_{2}(k)$ - неизвестные функции от показателя напряженного состояния $k$, которые зависят от свойств конкретного обрабатываемого материала.

При постоянных, на протяжении всего процесса деформирования, значениях показателя $\mu_{\sigma}=-1 ; 0 ;+1$ формула (10) может быть записана в виде:

$$
\begin{gathered}
\Lambda_{\mathrm{p}_{-1}}=a_{0}(\mathrm{k})-a_{1}(\mathrm{k})+a_{2}(\mathrm{k}), \\
\Lambda_{\mathrm{p}_{0}}=a_{0}(\mathrm{k}) \\
\Lambda_{\mathrm{p}_{+1}}=a_{0}(\mathrm{k})+a_{1}(\mathrm{k})+a_{2}(\mathrm{k}) .
\end{gathered}
$$

Опыт проведенных ранее исследований [19, 20, 29] показал, что при фиксированных значениях показателя $\mu_{\sigma}$ зависимость $\Lambda_{p}$ хорошо описывается функцией:

$$
\Lambda_{p}=\alpha \cdot \exp (\beta \cdot k)
$$

где $\alpha$ и $\beta$ - эмпирические коэффициенты. При постоянных за процесс значениях показателя $\mu_{\sigma}=-1 ; 0 ;+1$, формулу (12) запишем в виде: 
opter-accesss jürral

$$
\begin{aligned}
& \Lambda_{\mathrm{p}_{-1}}=\alpha_{1} \cdot \exp \left(\beta_{1} \cdot \mathrm{k}\right), \\
& \Lambda_{\mathrm{p}_{0}}=\alpha_{2} \cdot \exp \left(\beta_{2} \cdot \mathrm{k}\right), \\
& \Lambda_{\mathrm{p}_{+1}}=\alpha_{3} \cdot \exp \left(\beta_{3} \cdot \mathrm{k}\right) .
\end{aligned}
$$

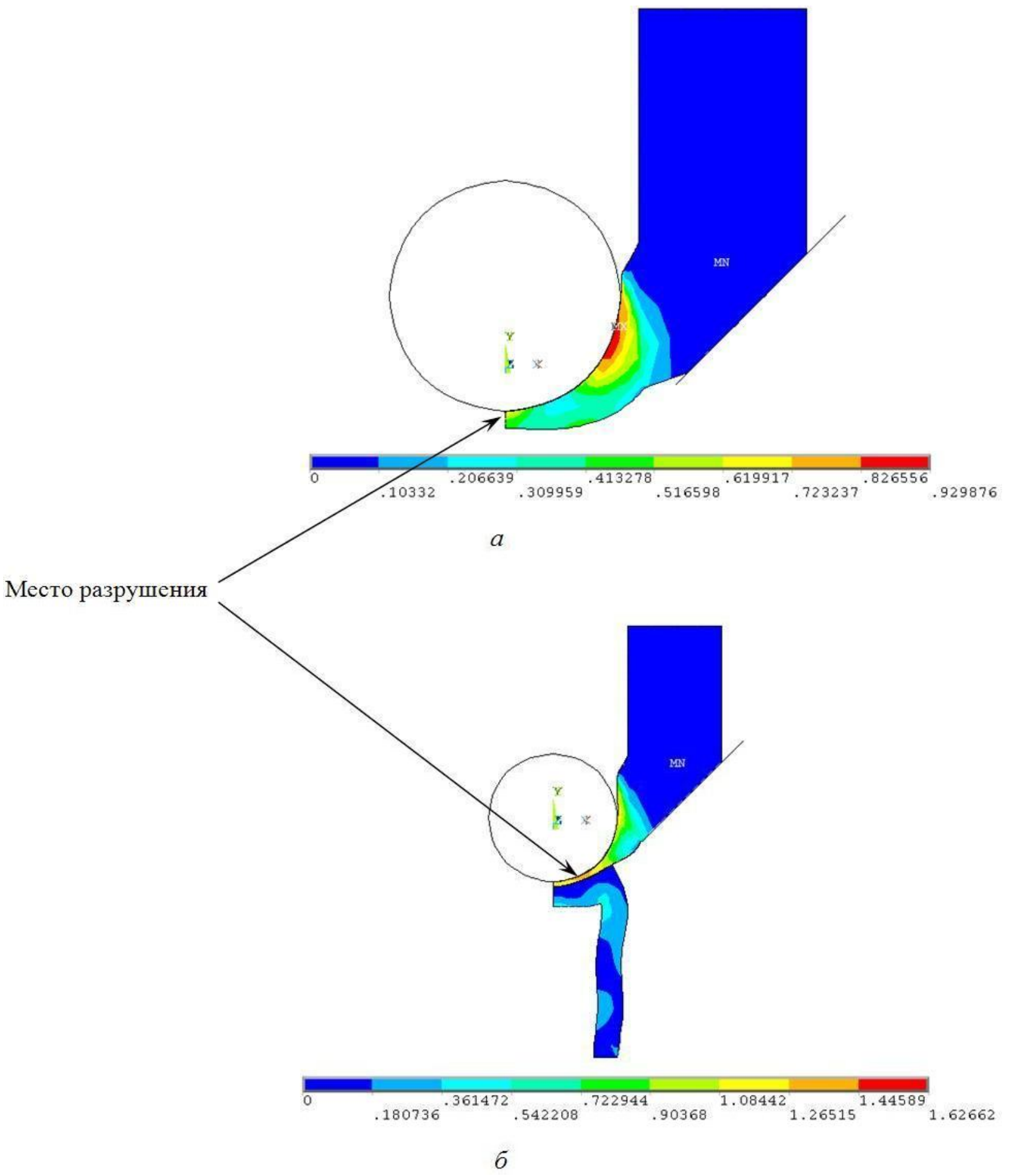

Рис. 9. Распределение эквивалентных пластических деформаций по сечению образца при выдавливании мембраны без подпора (a) и с подпором (б) 

уравнений:

Подставив правые части уравнений (13) в уравнения (11), получим систему

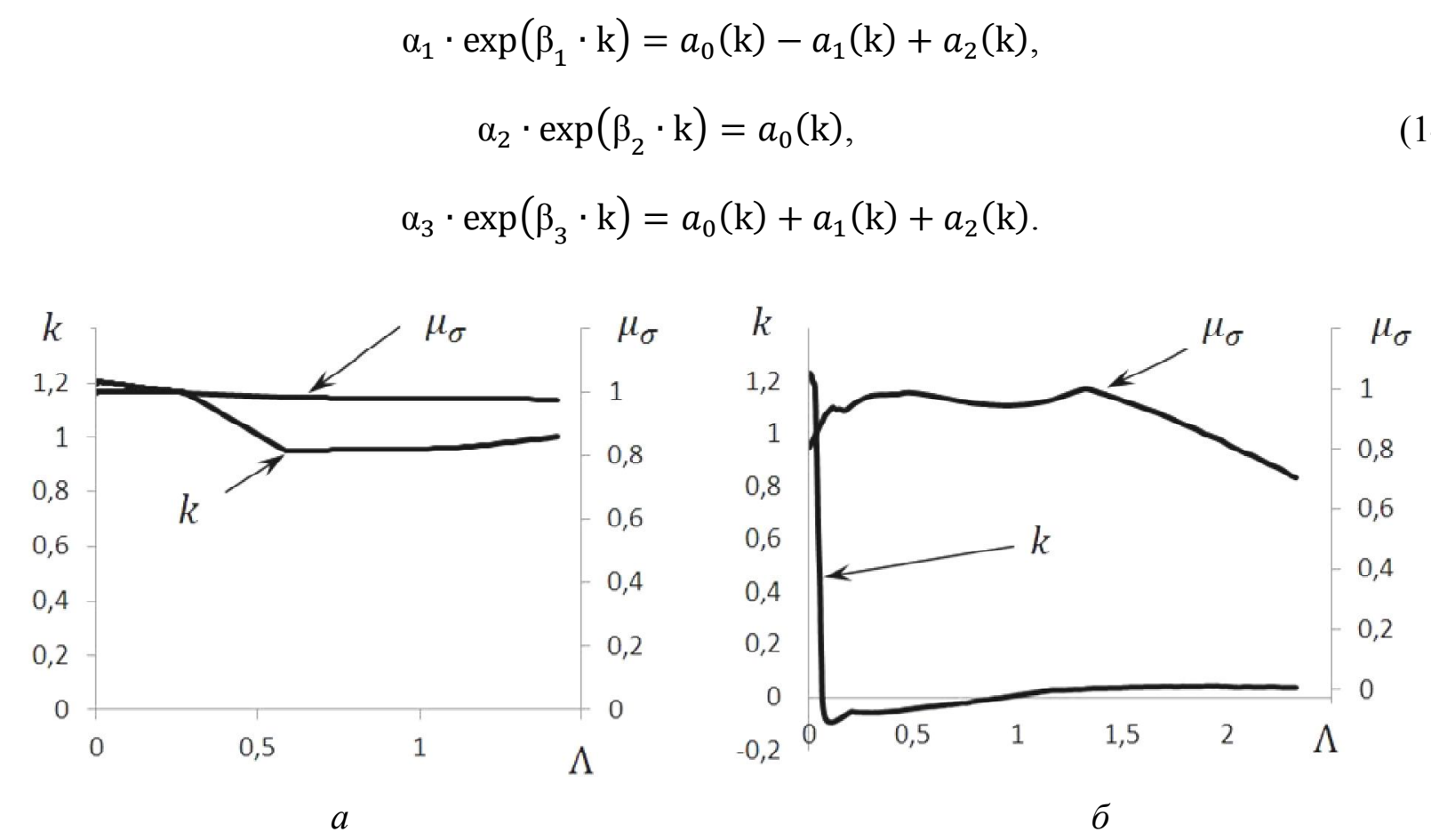

Рис. 10. Изменение показателей $k$ и $\mu_{\sigma}$ в процессе выдавливания мембраны без подпора $(a)$, выдавливания мембраны с подпором (б)

Решая систему уравнений (14), выразим функции $a_{0}(\mathrm{k}), a_{1}(\mathrm{k})$ и $a_{2}(\mathrm{k})$ через эмпирические коэффициенты $\alpha_{1}, \alpha_{2}, \alpha_{3}, \beta_{1}, \beta_{2}, \beta_{3}$ :

$$
\begin{gathered}
a_{0}(\mathrm{k})=\alpha_{2} \cdot \exp \left(\beta_{2} \cdot \mathrm{k}\right) \\
a_{1}(\mathrm{k})=\alpha_{3} \cdot \exp \left(\beta_{3} \cdot \mathrm{k}\right) / 2-\alpha_{1} \cdot \exp \left(\beta_{1} \cdot \mathrm{k}\right) / 2 \\
a_{2}(\mathrm{k})=\alpha_{3} \cdot \exp \left(\beta_{3} \cdot \mathrm{k}\right) / 2+\alpha_{1} \cdot \exp \left(\beta_{1} \cdot \mathrm{k}\right) / 2-\alpha_{2} \cdot \exp \left(\beta_{2} \cdot \mathrm{k}\right) .
\end{gathered}
$$

Подставив формулы (15) в уравнение (10), окончательно получим

$$
\begin{aligned}
\Lambda_{\mathrm{p}}= & \alpha_{2} \cdot \exp \left(\beta_{2} \cdot \mathrm{k}\right)+\left(\alpha_{3} \cdot \frac{\exp \left(\beta_{3} \cdot \mathrm{k}\right)}{2}-\alpha_{1} \cdot \frac{\exp \left(\beta_{1} \cdot \mathrm{k}\right)}{2}\right) \cdot \mu_{\sigma}+ \\
& +\left(\alpha_{3} \cdot \frac{\exp \left(\beta_{3} \cdot \mathrm{k}\right)}{2}+\alpha_{1} \cdot \frac{\exp \left(\beta_{1} \cdot \mathrm{k}\right)}{2}-\alpha_{2} \cdot \exp \left(\beta_{2} \cdot \mathrm{k}\right)\right) \cdot \mu_{\sigma}^{2} .
\end{aligned}
$$

Неизвестные коэффициенты $\alpha_{1}, \alpha_{2}, \alpha_{3}, \beta_{1}, \beta_{2}, \beta_{3}$, входящие в формулу (16), определяли путем минимизации функционала квадратичных отклонений значений поврежденности $\omega$ от значения условия разрушения $\omega=1$, рассчитанных для всех шести видов испытаний: 


$$
\sum_{i=1}^{6}\left(1-\omega_{i}\right)^{2} \rightarrow \min
$$

где $i$ - номер испытанного образца. Результаты испытаний приведены на рис. 1,4 и 10. Поврежденность $\omega_{i}$ рассчитывали по формуле (1). Варьирование неизвестных коэффициентов, входящих в формулу (16), осуществляли методом Хука - Дживса [30]. Для исключения попадания в локальный минимум функционала квадратичных отклонений (17) выбирали не одну начальную точку поиска, а некоторое их множество. Для каждой начальной точки поиска выполняли процедуру метода Хука - Дживса. При этом начальные значения неизвестных коэффициентов варьировали в интервале от -5 до 5 с шагом 0,5 . В результате получили значения неизвестных коэффициентов $\alpha_{1}=2,46 ; \alpha_{2}=2,38 ; \alpha_{3}=2,376 ; \beta_{1}=-0,089$; $\beta_{2}=-0,29 ; \beta_{3}=-0,678$.

На рис. 11 изображена диаграмма пластичности стали группы прочности X80. Диаграмма позволяет оценивать пластичность исследуемых материалов во всем интервале изменения показателя Лоде - Надаи. Из диаграммы видно, что пластичность стали неоднозначно зависит от вида напряженного состояния: в области растягивающих напряжений $(k>0)$ сталь наиболее пластична при деформации по схеме осесимметричного растяжения $\left(\mu_{\sigma}=-1\right)$, а в области сжимающих напряжений $(k<0)$ - при испытаниях на срез и выдавливание мембран. Этот факт свидетельствует о том, что стандартные испытания на растяжение $\left(\mu_{\sigma}=-1\right)$ и ударную вязкость $\left(\mu_{\sigma}=0\right)$ не позволяют адекватно оценить деформационную способность стали при всем разнообразии возможных условий работы магистрального трубопровода. Построение полной диаграммы пластичности позволяет значительно расширить сведения о деформационной способности материала.

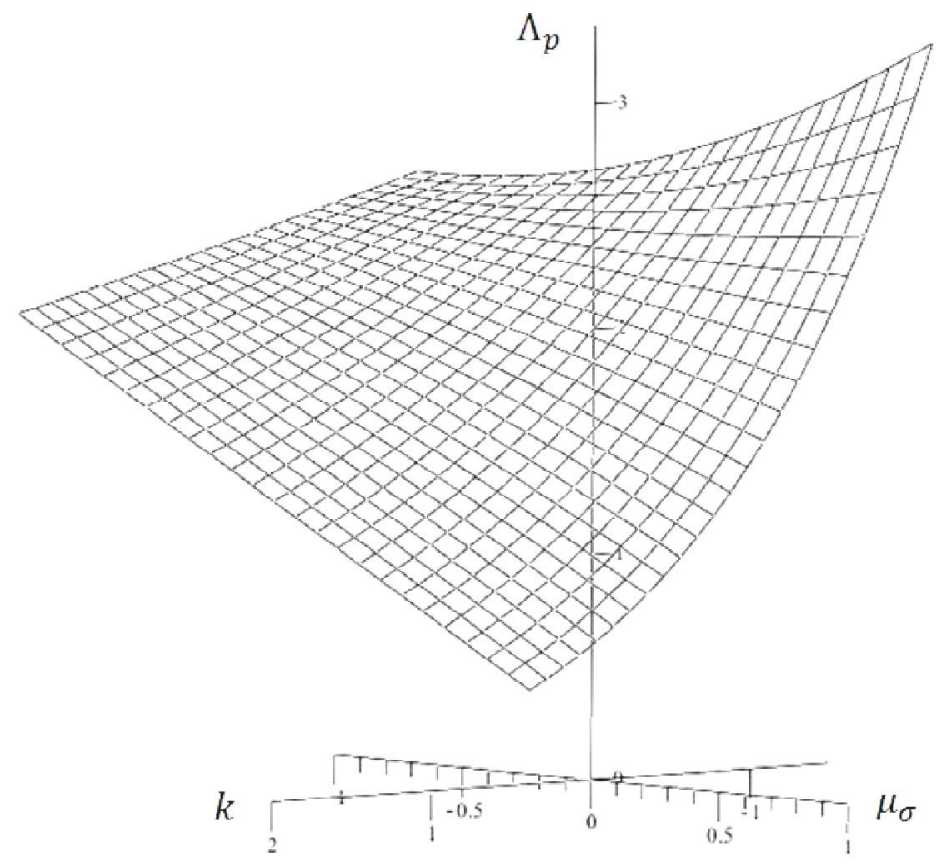

Рис. 11. Диаграмма пластичности стали группы прочности X80

\section{5 Возможное применение результатов исследования}

Покажем возможность использования полученной диаграммы пластичности на примере моделирования процесса ненормативного перемещения трубопровода [31]. Рассмотрен речной подводный участок магистрального трубопровода. Под воздействием различных природных факторов (подвижки и эрозия речного дна, размыв русла ит.п.) произошло размывание подводного трубопровода, в результате чего участок трубы оказался полностью в

Vichuzhanin D. I. et al. / The effect of the stress state on the ultimate plasticity of steel X80 
open-access journal

воде. При этом трубопровод всплыл под действием выталкивающей силы воды (рис. 12). Стрела арки подъема трубы достигла порядка 1000 мм. Моделирование осуществляли методом конечных элементов. Для материала трубы принимали изотропную упруго-пластическую модель деформационного упрочнения. В результате моделирования получено распределение поврежденности по сечению трубы (рис. 13), рассчитанной по формуле (1). В процессе деформирования на нижней стенке трубы образовалась складка, подобное явление наблюдалось в реальных экспериментах на изгиб трубы, выполненных в работе [32]. Видно, что после деформации трубопровода (даже с образованием складки) поврежденность по толщине стенки трубы имеет достаточно низкие значения, т.е. подобные деформации трубопровода не приводят к его разрушению. Аналогичные выводы сделаны в работе [31].

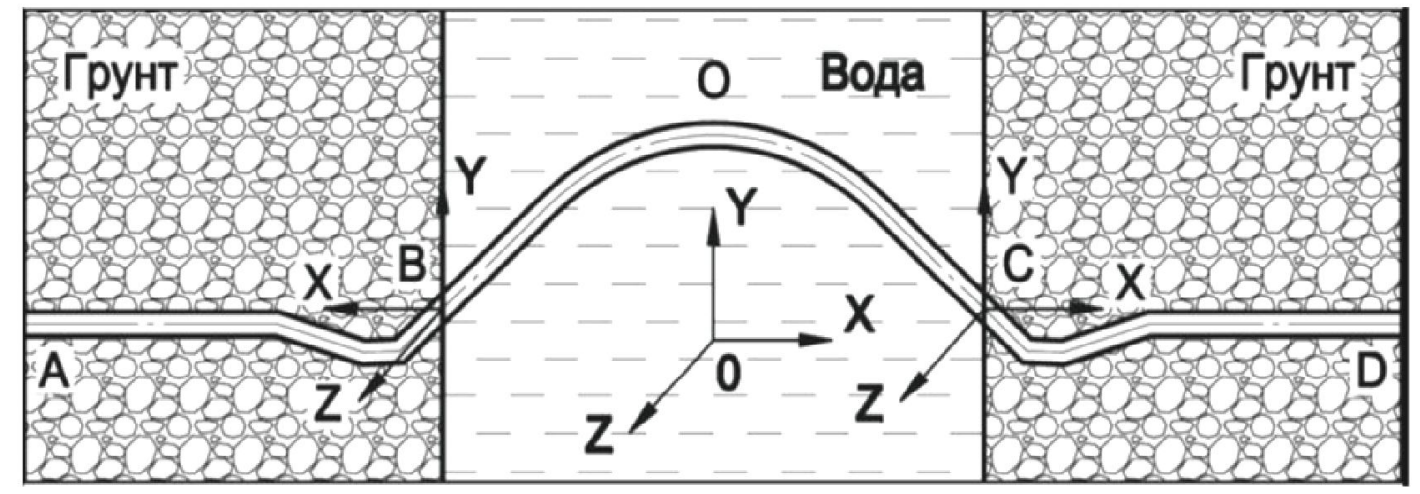

Рис. 12. Схема расположения трубопровода [31]

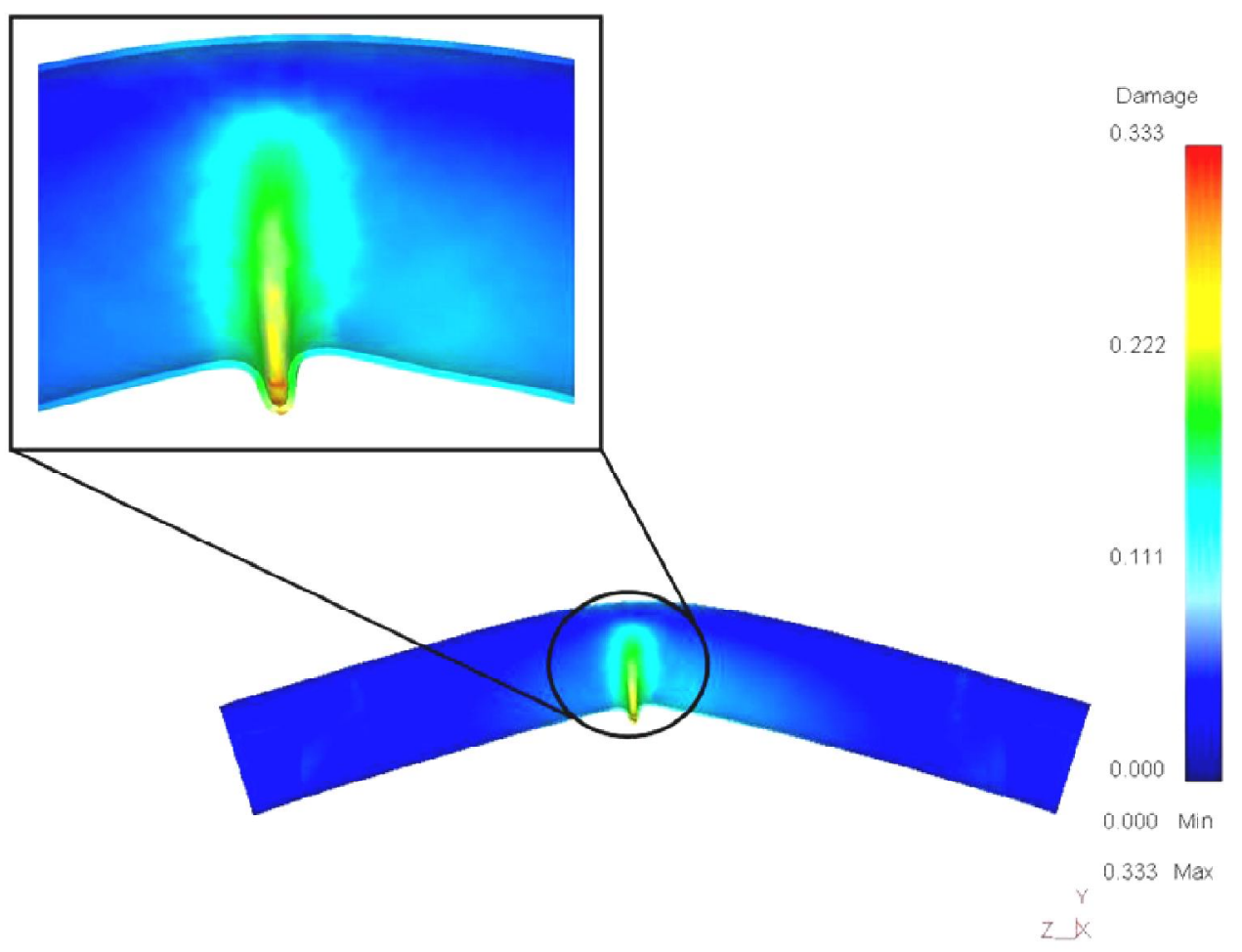

Рис. 13. Распределение поврежденности по сечению трубы магистрального трубопровода 


\section{4. Заключение}

Полученная диаграмма пластичности может быть использована для оценки ресурса пластичности стали группы прочности X80 в условиях сложного напряженнодеформированного состояния при экстремальных нагрузках магистральных трубопроводов.

Работа выполнена в соответствии с совместным проектом с СО РАН № 12-С-1-1030. Испытания проведены на оборудовании Центра коллективного пользования ИМАШ УрО PAH.

\section{Литература}

1. Obtaining high-quality properties of rolled material for large-diameter pipes based on formation of ferrite-bainite microstructure / Yu. D. Morozov, S. Yu. Nastich, M. Yu. Matrosov, O. N. Chevskaya // Metallurgist. - 2008. - Vol. 52, no 1-2. - P. 21-28.

2. Arabey A. B. Requirements on the metal in gas pipelines // Steel in translation. - 2010. Vol. 40, no 7. - P. 601-608.

3. Failure of pipe steel of X80 (K65) strength class / A. B. Arabey, I. Yu. Pyshmintsev, V. M. Farber, V. A. Khotinov, A. O. Struin // Steel in translation. - 2012. - Vol. 42, no 3. P. 212-218.

4. Микроструктура и дисперсные фазы в высокопрочных сталях газопроводных труб большого диаметра / А. Б. Арабей, В. М. Фарбер, И. Ю. Пышминцев, А. Г. Глебов, О. В. Селиванова, Н. В. Лежнин // Наука и техника в газовой промышленности. - 2011. - № 4. - С. 86-91.

5. Организация комплексных исследований отечественных труб для новых магистральных газопроводов нового поколения / В. В. Русакова, Т. П. Лобанова, А. Б. Арабей, И. Ю. Пышминцев и др. // Наука и техника в газовой промышленности. - 2009. № 1. - C. 17-21.

6. Structure and properties of low - carbon pipe steelafter pneumatic testing / I. Y. Pyshmintsev, A. N. Maltseva, A. M. Gervasev, M. A. Smirnov, A. V. Korznikov // Steel in translation. - 2011. Vol. 41, no 2. - P. 157-164.

7. Bridgman P. W. Studies in large plastic flow and fracture. - McGraw-Hill, 1952. - 362 p.

8. Atkins A. G. Fracture in forming // Journal of Materials Processing Technology. - 1996. Vol. 56. - P. 609-618.

9. Губкин С. И. Деформируемость металлов. - М.: Металлургиздат, 1953. - 199 с.

10. Смирнов - Аляев Г. А., Розенберг В. М. Теория пластических деформаций металлов. М.: Машгиз, 1956. - 367 с.

11. Bao Y., Wierzbicki T. On fracture locus in the equivalent strain and stress triaxiality space // International Journal of Mechanical Sciences. - 2004. - Vol. 46, iss. 1. - P. 81-98.

12. Experimental characterization and numerical modeling of micromechanical damage under different stress states / M. Achouri, G. Germain, P. Dal Santo, D. Saidane // Materials and Design. 2013. - Vol. 50. - P. 207-222.

13. Wierzbicki T., Xue L. On the effect of the third invariant of the stress deviator on ductile fracture // Impact \& Crashworthiness Laboratory. - 2005. - Report 136.

14. Smirnov S. V. Accumulation and healing of damage during plastic metal forming: simulation and experiment // Key Engineering Materials. - 2013. - Vol. 528. - P. 61-69.

15. Magnetic methods for estimation of load and damage levels in X70 steel / E. S. Gorkunov, S. Yu. Mitropolskaya, D. I. Vichuzhanin, E. A. Tueva // Physical Mesomechanics. - 2011. - Vol. 14, iss.1-2. - P. 85-93.

16. Качанов Л. М. О времени разрушения в условиях ползучести // Доклады Академии наук СССР, серия ОТН. 1958. - № 8. - С. 67-75.

17. Работнов Ю. Н. Вопросы прочности материалов и конструкций. - М.: АН СССР, 1959. - 120 с.

18. К Колмогоров В. Л. Напряжения, деформации, разрушение. - М.: Металлургия, 1970. - 229 с.

Vichuzhanin D. I. et al. / The effect of the stress state on the ultimate plasticity of steel X80 
19. Богатов А. А., Мижирицкий О. И., Смирнов С. В. Ресурс пластичности металлов при обработке давлением. - М.: Металлургия, 1984. - 144 с.

20. Смирнов С. В., Швейкин В. П. Пластичность и деформируемость углеродистых сталей при обработке давлением. - Екатеринбург: УрО РАН, 2009. - 256 с.

21. Cockcroft M. G., Latham D. J. Ductility and the workability of metals // Journal of the Institute of Metals. -1968 . - Vol. 96. - P. 33-39.

22. Gurson A. L. Continuum theory of ductile rupture by void nucleation and growth: Part I. Yield critetria and flow rules for porous ductile media // Journal of Engineering Materials and Technology. - 1977. - Vol. 99. - P. 2-15.

23. Johnson G. R., Cook W. H. Fracture characteristics of three metals subjected to various strains, strain rates, temperatures and pressures // Engineering Fracture Mechanics. - 1985. - Vol. 21 (1). - P. 31-48.

24. Lemaitre J. A Course on Damage Mechanics. - Berlin: Springer-Verlag, 1992. - 44 p.

25. Bai Y., Wierzbicki T. A new model of metal plasticity and fracture with pressure and Lode dependence // International Journal of Plasticity. - 2008. - Vol. 24, iss. 6. - P. 1071-1096.

26. Bammann D. J., Solanki K. N. On kinematic, thermodynamic, and kinetic coupling of a damage theory for polycrystalline material //International Journal of Plasticity. - 2010. - Vol. 26, iss. 6. - P. 775-793.

27. Davidenkov N. N., Spiridonova N. I. Mechanical methods of testing analysis of the state of stress in the neck of a tension test specimen // Proc. ASTM. - 1946. - Vol. 46. - P. 1147.

28. Контактное трение в процессах обработки металлов давлением / А. Н. Леванов, В. Л. Колмогоров, С. П. Буркин и др. - М.: Металлургия, 1976. - 416 с.

29. Smirnov S. V., Domilovskaya T. V., Bogatov A. A. In: Advanced Methods in Materials Processing Defects, by M. Predeleanu and P. Gilormini // Elsivier Science B.V. - 1997. - P. 71-80.

30. Hooke R., Jeeves T. A Direct Search Solution of Numerical and Statistical Problems // Journal of the ACM. - 1961. - Vol. 8. - P. 212-229.

31. Исламгалеева Л. Ф., Зарипов Р. М. Влияние степени обводнения грунта прилегающих подземных участков на напряженно - деформированное состояние подводного газопровода [Электронный ресурс] // Электронный научный журнал «Нефтегазовое дело». - 2011. - № 6. - C. 116-129. URL: http://www.ogbus.ru/authors/Islamgaleeva/Islamgaleeva 1.pdf (дата обращения: 21.07.2014).

32. Suzuki N., Kondo J., Shimamura J. Strain Capacity of High - Strength Line Pipes // JFE Technical Report. - 2008. - no. 12. - P. 20-26. 